\title{
Three Phase Resolution Transmitarray Element for Electronically Reconfigurable Transmitarrays
}

\author{
Nguyen Minh Thien, Nguyen Huu Minh, Nguyen Binh Duong \\ International University, Ho Chi Minh City, Vietnam \\ Correspondence: Nguyen Binh Duong, nbduong@hcmiu.edu.vn \\ Communication: received 27 November 2019, revised 30 December 2019, accepted 30 December 2019 \\ Digital Object Identifier: 10.32913/mic-ict-research.v2019.n2.905 \\ The Editor coordinating the review of this article and deciding to accept it was Prof. Nguyen Tan Hung
}

\begin{abstract}
Electrical beam scanning is a feature enabling an antenna array to electrically control its main beam toward a desired direction. In this paper, a three-phase state element for electronically reconfigurable transmitarrays is presented. The element is made up of $\mathrm{C}$-patches and modified ring slots loaded rectangular gaps. By controlling the bias state of four p-i-n diodes, three phase states are obtained. The dimension of the element is optimized by using full-wave EM simulation and performance of the element is validated by both simulation and an experimental waveguide system. A transmitarray consisting of $12 \times 12$ elements has been simulated to validate the steering capabilities. Experimental results indicate the element has good characteristics and excellent phase change capabilities.
\end{abstract}

Keywords: Transmitarray antenna, flat lens antenna, reconfigurable transmitarray antenna.

\section{INTRODUCTION}

Planar array antennas have been widely used in many applications requiring low profile antenna with high gain and beam control capacity. Examples of radar applications working at X-band are marine rain map radar, airborne weather surveillance and warning system and Synthetic Aperture Radar (SAR) for satellites. Recently, modern tunable electronic components enable high-speed beam forming and vibration elimination for planar array antennas. This is a considerable advantage over the traditional high gain antennas in which a bulky mechanical system is required to physically rotate the antenna direction. Phased array, reflectarray and transmitarray are three types of antennas that can provide high gain. A planar phased array typically uses a microstrip feeding network to excite the array elements in a specified state to form the main beam. On the other hand, reflectarray and transmitarray utilize

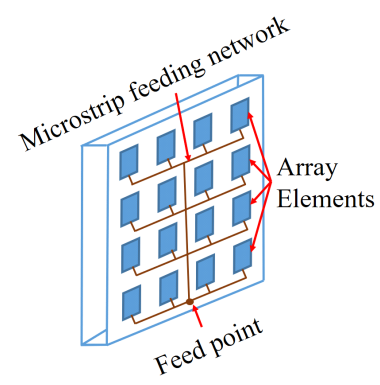

(a)

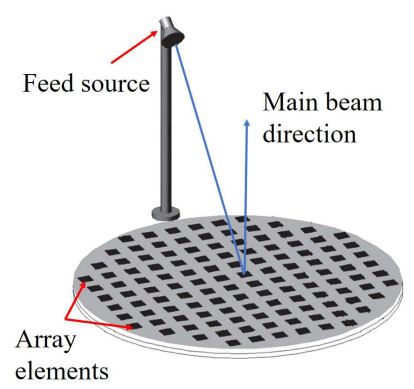

(b)

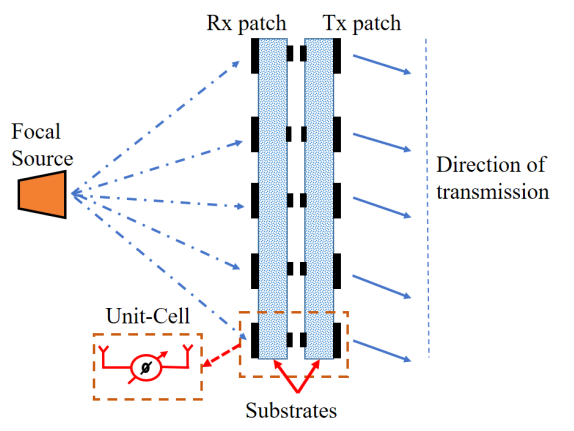

(c)

Figure 1. Basic diagram of (a) planar phased array, (b) reflectarray, and (c) transmitarray.

the space-feed mechanism in which an external feed source is placed at a focal point, thus no complex beamforming network is required in the array's surface. A transmitarray, as shown in Figure 1(c), overcomes the disadvantage of reflectarray which is the feed blockage phenomenon due to the fact that the feed source of transmitarray is located in the opposite side of the radiated wave. Recent transmitarray designs are mainly based on multilayer frequency selective surfaces (M-FSS) [1-5]. A typical transmitarray using MFSS structure composes of multiple microstrip arrays of 
antenna elements and a feed source. The first planar layer that is placed in front of the feed source is labeled as receiver $(\mathrm{Rx})$, working in receiving mode. The last layer acts as transmitter (Tx).

A transmitarray transforms the spherical wave from the feed source into a planar wavefront. In order to collimate the energy toward a specific direction, each element is designed to provide a desired phase shift to compensate the phase error due to different path lengths from the feed source. Hence, the performance of the element is the utmost factor in any transmitarray design. The transmitarray element must be able to not only generate desired phase shift but also to achieve minimum energy loss.

To achieve electronically beam-scanning ability, recent works have proposed different designs for transmitarrays in which different tunable components are employed to control the main beam direction. The transmitarrays proposed in [6-8] use varactors. The advantage of a transmitarray element using varactors is the continuous phase control. This is based on the fact that the capacitance of varactors is varied as a function of the DC bias voltage. Different capacitance values could lead to different transmission phase states of an element. However, to achieve enough phase range for implementing a transmitarray, transmitarray elements proposed in [6-8] use a large number of varactors that leads to high losses and increases the complexity of the element geometry.

Besides the electronically reconfigurable transmitarray based on varactors, various transmitarrays using RF switches such as RF-MEM [9], p-i-n diodes [10-13] have been proposed. Although RF switches cannot provide a continuous phase variation, they are much more suitable for transmitarrays operating at high frequencies, especially in millimeter-wave frequency band. Moreover, the RF switches are not much sensitive to DC voltage, this makes the reconfigurable transmitarrays using RF-switches more stable against the fluctuation of DC bias voltage. In [10-12], an 1-bit reconfigurable transmitarray element using two $\mathrm{p}-\mathrm{i}-\mathrm{n}$ diodes and in [13], a 2-bit reconfigurable transmitarray element have been proposed. The disadvantage of the reconfigurable transmitarray using RF switches is the low phase resolution. A low phase resolution leads to large quantization loss, resulting in gain degradation of the transmitarray. To increase phase resolution, higher number of switches is required. However, this may increase the complexity of the biasing network. In design of an electronically reconfigurable transmitarray using RF-switches, there is a trade-off between the number of phase states and the complexity of the transmitarray element.

In this paper, a reconfigurable transmitarray with threephase states is presented. The three phase states are ob-

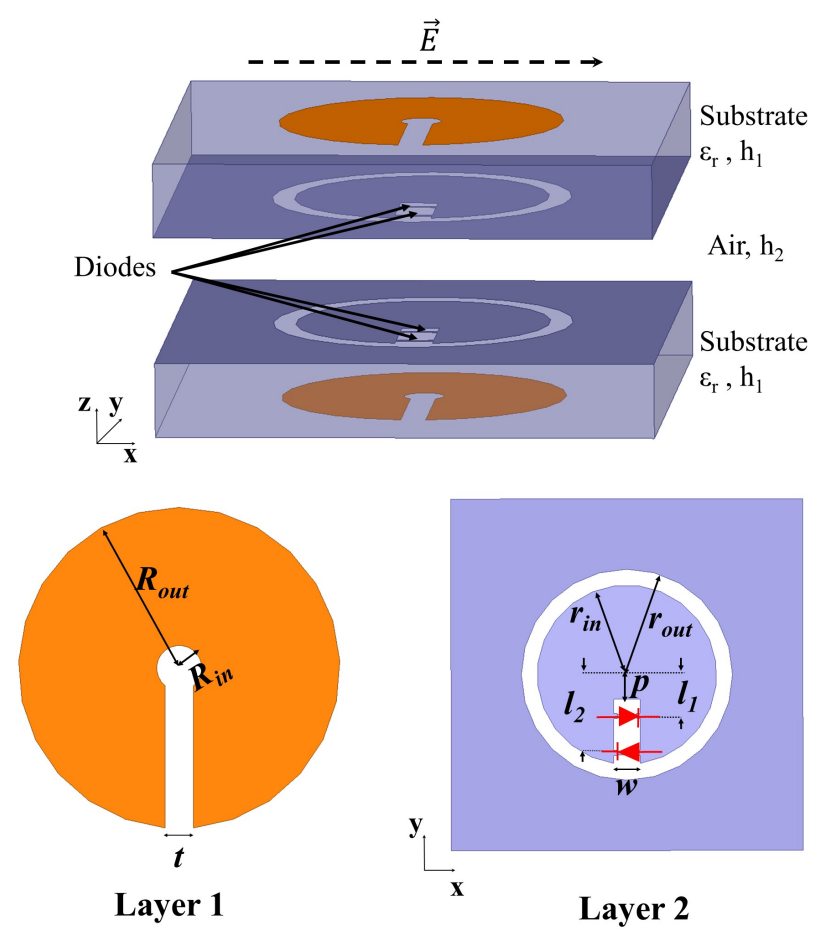

Figure 2. Geometry of the proposed transmitarray element.

tained by using four $\mathrm{p}-\mathrm{i}-\mathrm{n}$ diodes. The proposed element is an extended version of our previous work [12] for the purpose of increasing the number of phase states of the transmitarray element. Four additional p-i-n diodes are biased in pairs, which makes the biasing network simple. The three-phase state element is designed to operate at the center frequency of $11.5 \mathrm{GHz}$. A passive prototype with the use of an ideal switch has been fabricated. Measurements on the prototype have been conducted to demonstrate the performances of the proposed element. A transmitarray of $12 \times 12$ elements was designed and simulated to validate the performance and the capability of main beam reconfigurability.

\section{Transmitarray Element Design}

\section{Element Design}

Figure 2 shows the proposed transmitarray element that is implemented using four metallic layers printed on two identical Roger RO5870 substrates. The Roger RO5870 substrate has a thickness of $1.575 \mathrm{~mm}$ and dielectric constant $\varepsilon_{r}=2.33$. Two substrates are separated by $1.5 \mathrm{~mm}$. On each substrate, a C-patch and a modified ring slot loaded by a rectangular gap are printed. The C-patch is on the top of the substrate and the modified ring slot loaded by a rectangular gap is on the bottom of the substrate. Two p$\mathrm{i}$-n diodes are inserted on the rectangular gap of each ring slot. The p-i-n diodes are used to control the transmission 


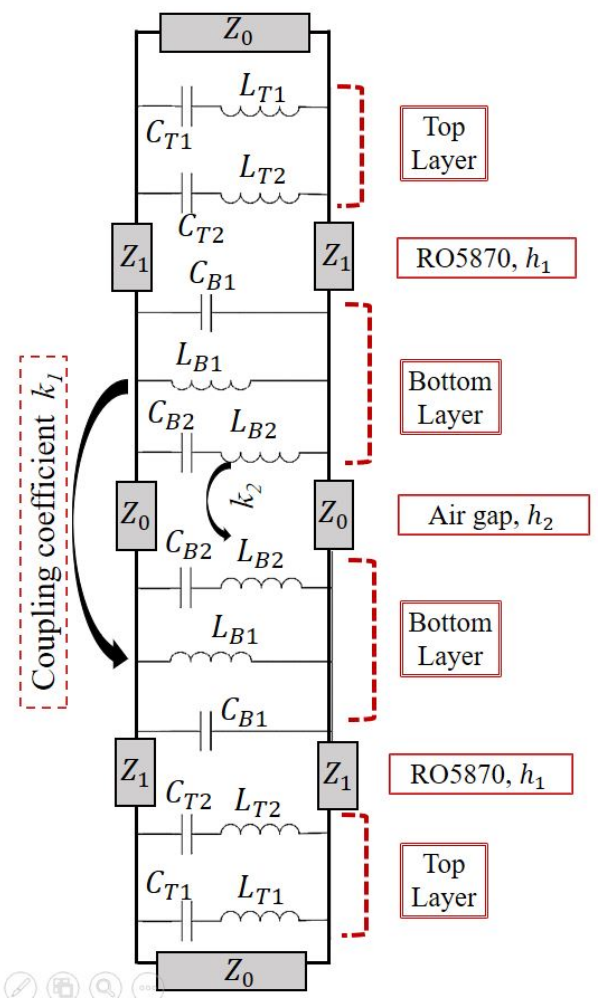

Figure 3. Equivalent circuit of our proposed structure.

phase shift. The element is designed to operate at $\mathrm{X}$ band with the center frequency of $11.5 \mathrm{GHz}$. It operates with linear polarization where E-field of the incident wave is perpendicular to the gap of the C-patch. The cell size is 14 $\mathrm{mm}$, equivalent to $0.5 \lambda_{o}$ where $\lambda_{o}$ is the wavelength in the free space.

The proposed element is developed from the transmitarray element that was investigated in [12]. In this work, two more diodes are added to provide the third phase state. Increasing the phase states is based on the ability of changing the transmission phase by modifying the length of the rectangular gap of the ring slot. Therefore, we can increase the number of phase states by increasing the number of switches on the rectangular gap to control the length of the rectangular gap. To understand the working principle of the proposed structure as well as the capability of changing the phase according the length of the rectangular gap of the ring slot, it is helpful to analyze the equivalent circuit of the structure. As presented in [14], the equivalent circuit of the element can be represented as shown in Figure 3.

For the equivalent circuit, the two C-patches placed on the top of two substrates are modeled as a parallel circuit containing two series LC tanks $\left(C_{T 1}, L_{T 1}, C_{T 2}, L_{T 2}\right)$. The ring slot loaded with a rectangular gap is represented by a parallel $\mathrm{LC}$ tank $\left(C_{B 1}, C_{B 1}\right)$ placed in parallel with a series LC tank $\left(C_{B 2}, L_{B 2}\right)$. The substrate with a thickness
TABLE I

DIMENSIONS OF THE PROPOSED ELEMENT

\begin{tabular}{|c|c|}
\hline Element & Dimensions $(\mathbf{m m})$ \\
\hline Layer 1 & $R_{\text {in }}=0.8 ; R_{\text {out }}=5.7 ; t=1$. \\
\hline Layer 2 & $\begin{array}{c}R_{\text {in }}=5.1 ; R_{\text {out }}=6 ; w=1.5 ; \\
p=1.4 ; l_{1}=2.5 ; l_{2}=4.3 .\end{array}$ \\
\hline Substrates & $h_{1}=1.575$ \\
\hline Air gap & $h_{2}=1.5$ \\
\hline
\end{tabular}

of $h_{1}$ is modeled as a transmission line with a length of $h_{1}$ and a characteristic impedance of $Z_{1}=Z_{0} / \sqrt{\varepsilon_{r}}$, where $\varepsilon_{r}$ is the relative permittivity of the substrate and $Z_{0}=377 \Omega$ is the free space impedance. As shown in Figure 3, the element structure has multi-resonances. For a transmitarray element structure, multi-resonances can allow a large phase range $[1,2]$. The modification of a ring slot to become the ring slot loaded with a rectangular gap makes the structure having two resonances. The original ring slot creates a single resonance that can be modelled as a parallel LC tank $\left(C_{B 1}, L_{B 1}\right)$. Adding a rectangular gap creates the second resonance at a high frequency. It is modeled by a series LC tank $\left(C_{B 2}, L_{B 2}\right)$. The frequency of the second resonance is a function of the length of the rectangular gap. The second resonant frequency is shifted towards to the lower frequency when the length of the rectangular gap is increased. Variation of the second resonance of the ring slot leads to a variation of the transmission phase of our structure. Therefore, three phase states can be achieved by using four $\mathrm{p}-\mathrm{i}-\mathrm{n}$ diodes. These four $\mathrm{p}-\mathrm{i}-\mathrm{n}$ diodes are placed on the rectangular gaps to control the length of the rectangular gaps. We use the same biasing circuit as presented in [12] to supply the DC power to $\mathrm{p}-\mathrm{i}-\mathrm{n}$ diodes. Four diodes work in pairs. Each pair is biased in reverse compared to the other. The position of four diodes is optimized to obtain three phase states with a step of $120^{\circ}$ at $11.5 \mathrm{GHz}$ according to ON/OFF state of diodes. The first phase state is obtained when all diodes are OFF. The second phase state and the third phase state are obtained when the first pair and second pair of diodes are turned ON, respectively. The parameters of the element are shown in Table I.

\section{Frequency Response of The Transmitarray Element}

The performance of the element should be validated before implementing a transmitarray. ANSYS HFSS software version 13 is used to simulate and to optimize the proposed element. To obtain the transmission phase and magnitude, a method is to use the waveguide simulator. Since the center 
frequency of the element is $11.5 \mathrm{GHz}$, the WR-90 standard waveguide is suitable to be used as waveguide simulator. In the simulation, the element is placed in the open-end of two WR-90 standard waveguides. Two excitation ports are assigned at the other ends of two waveguides to measure the transmission coefficients. Before the final version of an electronically reconfigurable transmitarray is implemented, the performance of the element is first evaluated using ideal RF-switches. In this case, metallic strips are used as ideal pi-n diodes. For the ON state of a diode, the metallic strips are inserted on the gaps. For the OFF state, the metallic strips are removed.

Figure 4 shows the simulated transmission coefficients of the proposed element for three phase states. As it can be seen, the transmission magnitude of three phase states at $11.5 \mathrm{GHz}$ is greater than $-1 \mathrm{~dB}$. The common $-3 \mathrm{~dB}$ transmission bandwidth of three phase states is $16.5 \%$ from $10.6 \mathrm{GHz}$ to $12.5 \mathrm{GHz}$. As shown in Figure 4(b), the transmission phase curve successfully changes when we change the state of four diodes. Three phase curves have a step of $120^{\circ}$ at $11.5 \mathrm{GHz}$. However, the step of $120^{\circ}$ is not maintained for frequencies far from $11.5 \mathrm{GHz}$, due to the non-linearity of the phase curves.

\section{Experimental Validation of the Element}

A prototype of the element is implemented to validate the performance of the proposed element. The element is fabricated by standard PCB fabrication technique. A small metallic strip that acts as an ideal switch in the ON state is soldered across the rectangular gap of ring slot layer. That metallic strip is removed for the OFF state of the switch, as shown in Figure 5.

The method to measure the frequency response of the element prototype is also to use waveguide simulators. This technique requires two WR-90 standard waveguides whose open-end size is $22.86 \times 10.16 \mathrm{~mm}^{2}$. Since the element's shape is a square while the aperture of the waveguide is rectangular, two rectangular-to-square transitions are implemented and they are used as an adaptor to put the element in the middle of two waveguides. A metallic plate with a hollow of $14 \times 14 \times 1.5 \mathrm{~mm}^{3}$ is inserted between two parts of the element to ensure that two substrates are separated by an air gap of $1.5 \mathrm{~mm}$. Figure 6 presents the measurement system. The measurement of the transmission coefficients is performed using Agilent E5071C Vector Network Analyzer. The measurement system has been calibrated at the ends of two straight waveguides, not including the two rectangular-to-square transitions. Figure 7 shows the measured transmission coefficients in comparison with that of the simulation. As shown in Figure 7, the measured results agree well with simulated results.

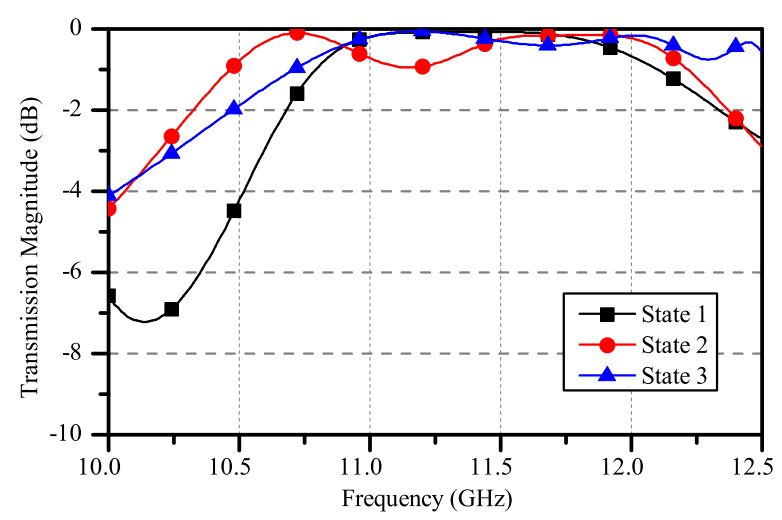

(a)

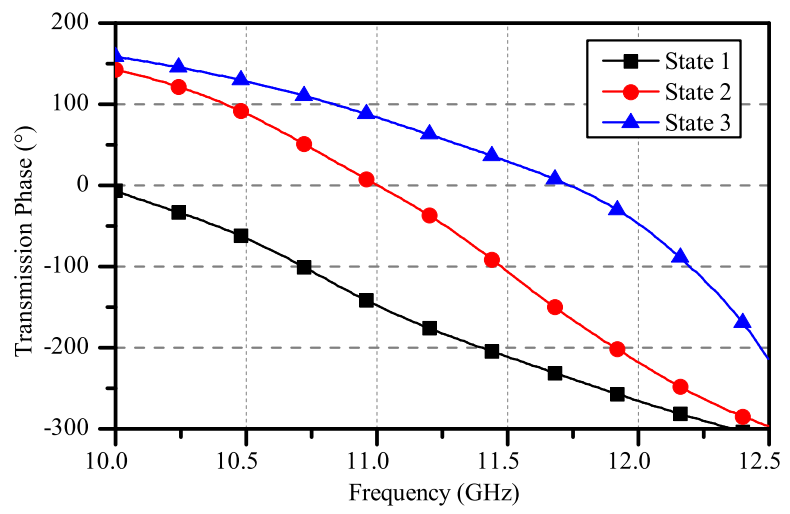

(b)

Figure 4. (a) Simulated transmission magnitude and (b) transmission phase of the proposed element.

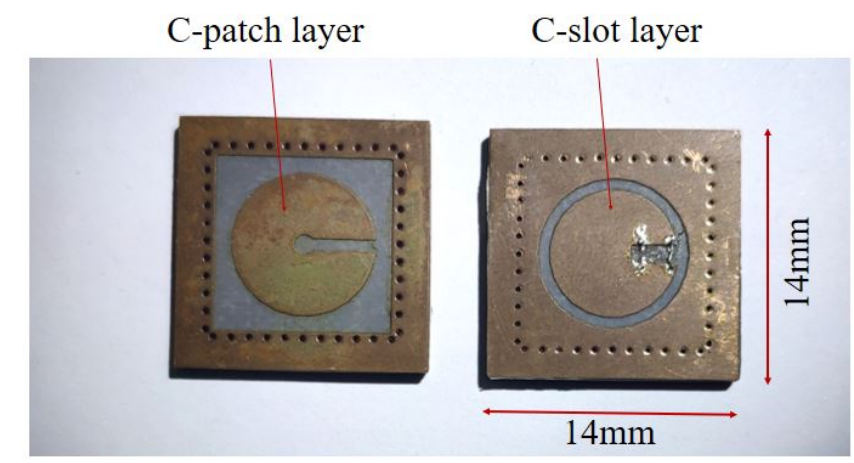

Figure 5. (Simulated transmission magnitude (left) and transmission phase (right) of the proposed element.

\section{Transmitarkay Design}

A square transmitarray antenna is designed with $12 \times 12$ elements to validate the radiation characteristics and beam steering capacity. As the periodicity of each element is 14 $\mathrm{mm}$, the transmitarray size is $168 \times 168 \mathrm{~mm}^{2}$, corresponding to $6.44 \lambda_{o} \times 6.4 \lambda_{o}$ at $11.5 \mathrm{GHz}$. A small aperture horn antenna is used as the feed source for the array. Its aperture is $32 \times 23 \mathrm{~mm}^{2}$ and its directivity is $11 \mathrm{~dB}$. The horn antenna is placed at a focal length of $150 \mathrm{~mm}$ corresponding 


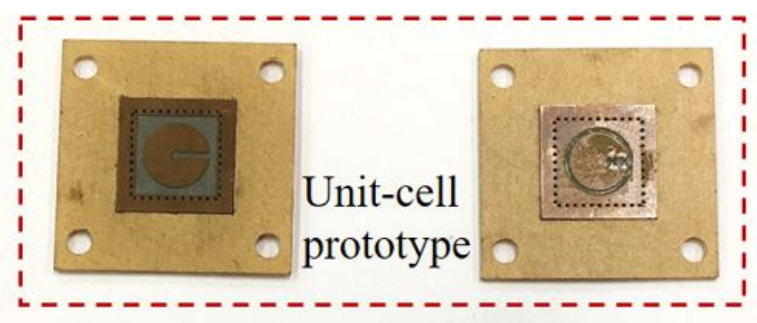

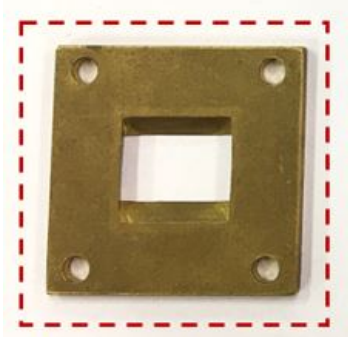

Rectangular-tosquare transition

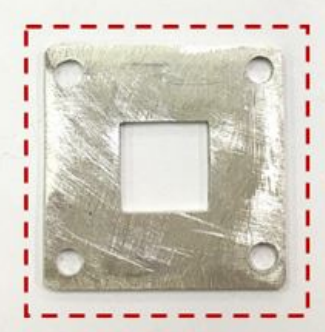

Metallic plate

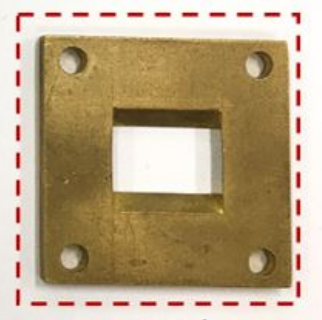

Rectangular-tosquare transition

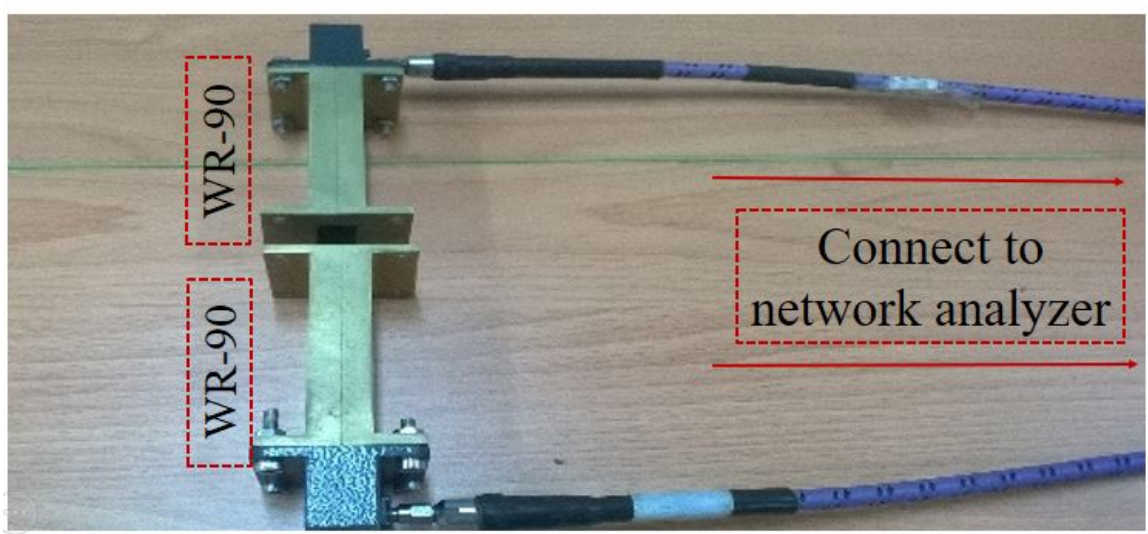

Figure 6. The measurement system.

to an F/D ratio of 0.89 . The transmitarray in $3 \mathrm{D}$ and the simulation environment are shown in Figure 8.

In the design of a space-fed array antenna, to steer the main beam to direction $(\theta, \phi)$, the transmission phase of each element can be calculated using equations (1) and (2) as follows:

$$
\begin{aligned}
& \varphi\left(x_{i}, y_{i}\right)=k_{0}\left(d_{i}-\sin \theta\left(x_{i} \cos \phi+y_{i} \sin \phi\right)\right), \\
& d_{i}=\sqrt{\left(x_{i}-x_{f}\right)^{2}+\left(y_{i}-y_{f}\right)^{2}+\left(z_{i}-z_{f}\right)^{2}},
\end{aligned}
$$

where $(\theta, \phi)$ is the direction of main beam, $x_{i}, y_{i}$ and $z_{i}$ are the coordinates of the $i^{\text {th }}$ element, $x_{f}, y_{f}$ and $z_{f}$ are the coordinates of the feed source, and $k_{0}$ is a propagation constant.

According to equation (1), the phase distribution on the transmitarray aperture is depicted in Figure 9. In this figure, the desired main beam direction is $\theta=\phi=0^{\circ}$.
Since the transmitarray antenna is based on the element which provides three phase states as discussed above, after calculating the theoretical compensation phase of each element using equations (1) and (2) for a main beam at direction $(\theta, \phi)$, the real phase $\psi\left(x_{i}, y_{i}\right)$ of the element at the position with the coordinates $x_{i}, y_{i}$ on the transmitarray is quantized using equation (3). This corresponds to the three phase states:

$$
\psi\left(x_{i}, y_{i}\right)= \begin{cases}0^{\circ}, & -60^{\circ}<\varphi\left(x_{i}, y_{i}\right)<-60^{\circ}, \\ 120^{\circ}, & 60^{\circ}<\varphi\left(x_{i}, y_{i}\right)<180^{\circ}, \\ 240^{\circ}, & 180^{\circ}<\varphi\left(x_{i}, y_{i}\right)<300^{\circ},\end{cases}
$$

where $\psi\left(x_{i}, y_{i}\right)$ is the quantized phase of the $i^{\text {th }}$ element at the position with the coordinates $x_{i}, y_{i}$.

In order to evaluate the beam steering capabilities of the transmitarray, various phase distributions obtained by arranging the suitable transmission phase are designed. 


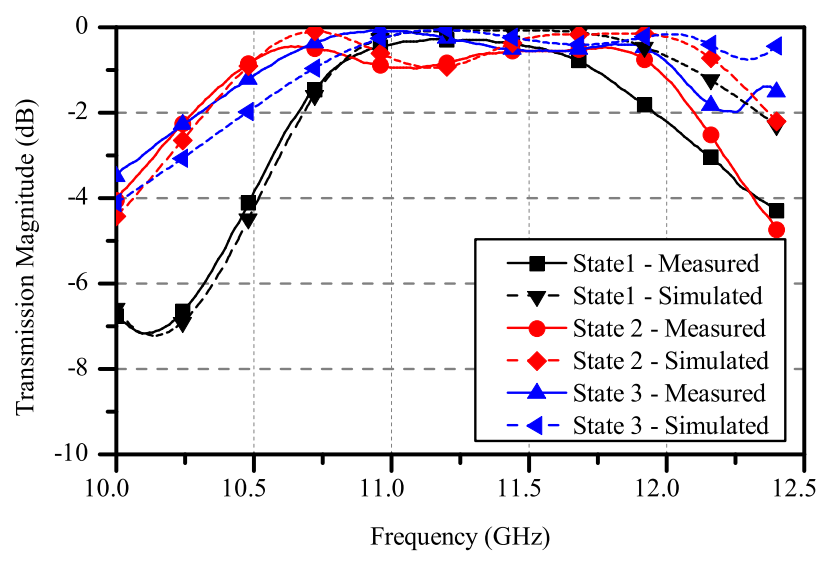

(a)

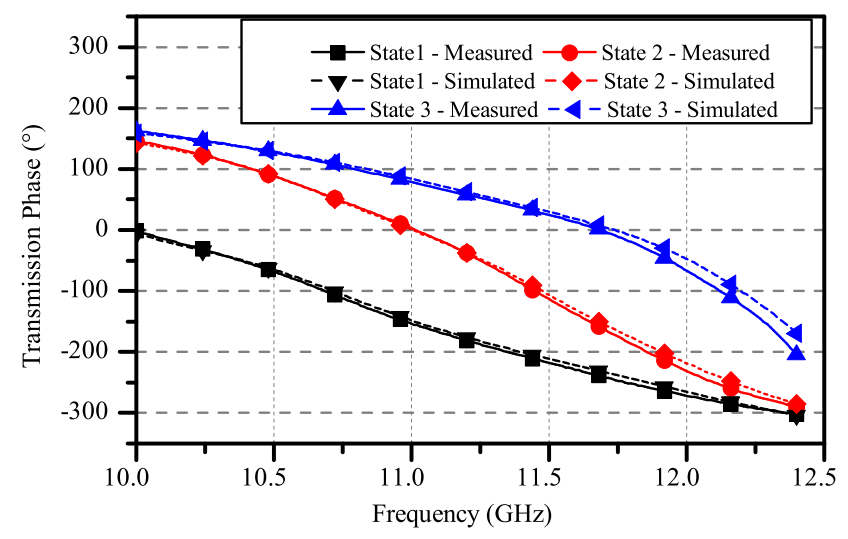

(b)

Figure 7. Measured and simulated transmission coefficients of the prototype: (a) transmission magnitude and (b) transmission phase.

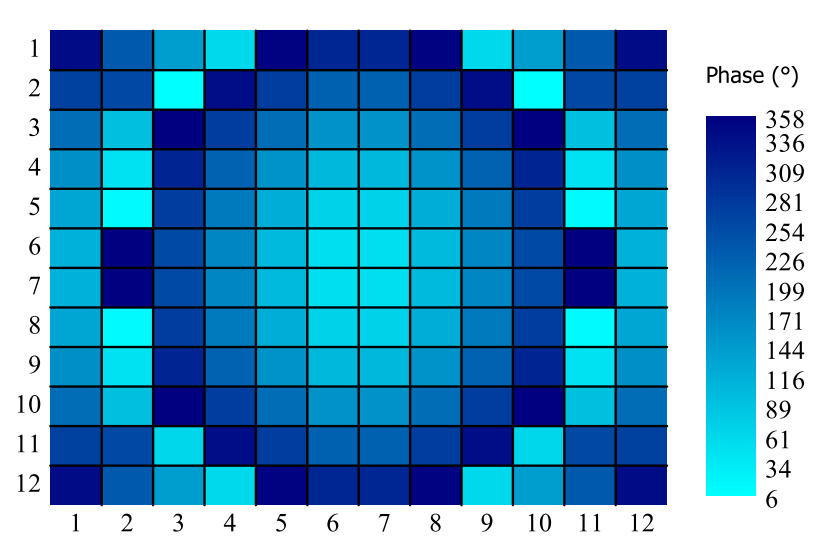

Figure 9. Theoretical compensation phase distribution required in the broadside transmitarray.

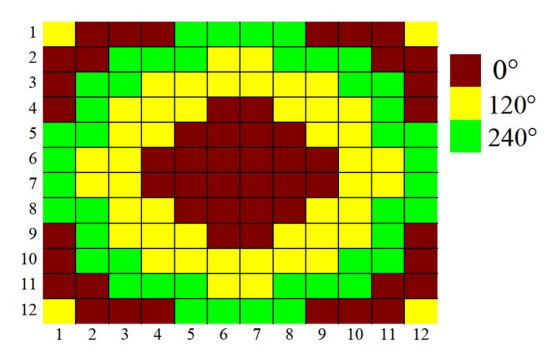

(a)
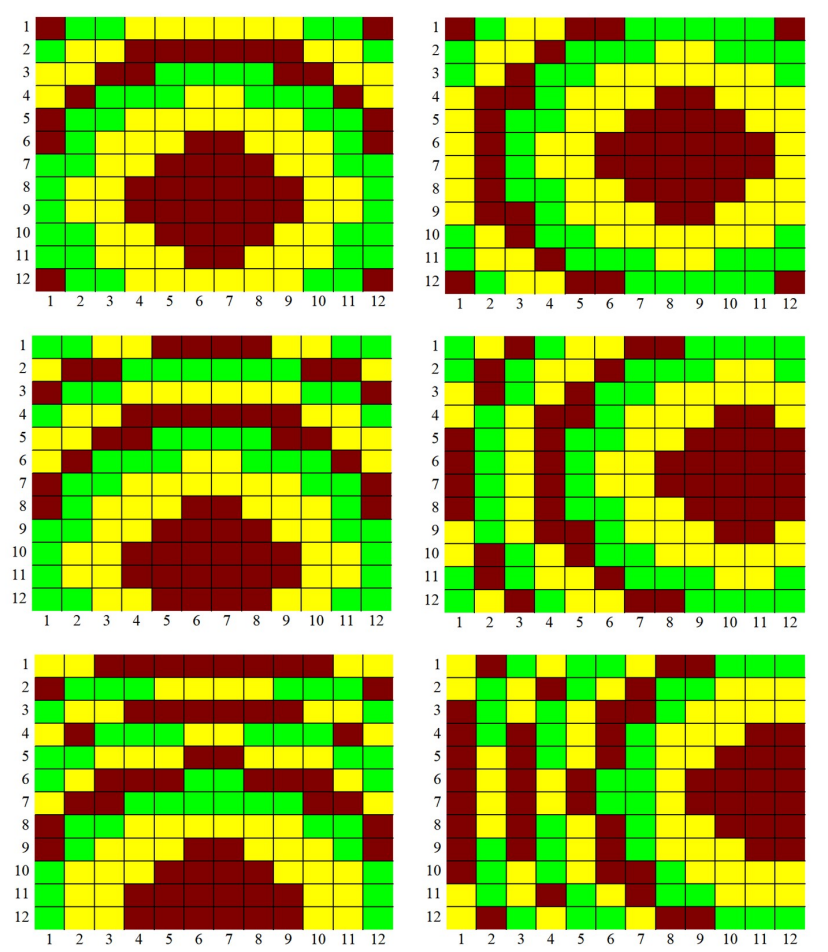

(b)

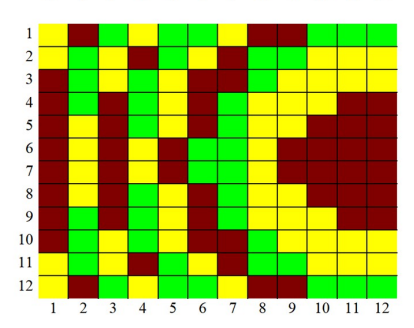

(c)

Figure 10. Phase distribution for the main beam pointed at different angles: (a) $\theta=0^{\circ}, \phi=0^{\circ}$ or $90^{\circ}$, (b) $\phi=0^{\circ}, \theta=10^{\circ}, 20^{\circ}, 30^{\circ}$, and (c) $\phi=90^{\circ}, \theta=10^{\circ}, 20^{\circ}, 30^{\circ}$.

Figure 8. Simulation system for $12 \times 12$-element transmitarray. 


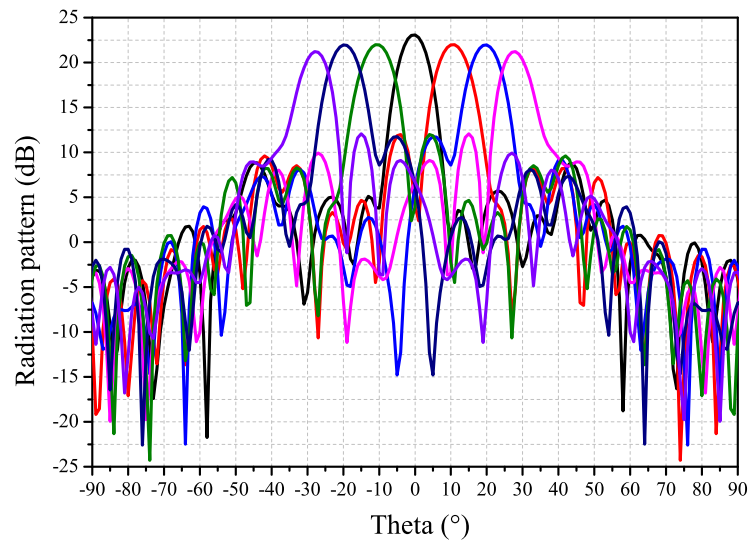

(a)

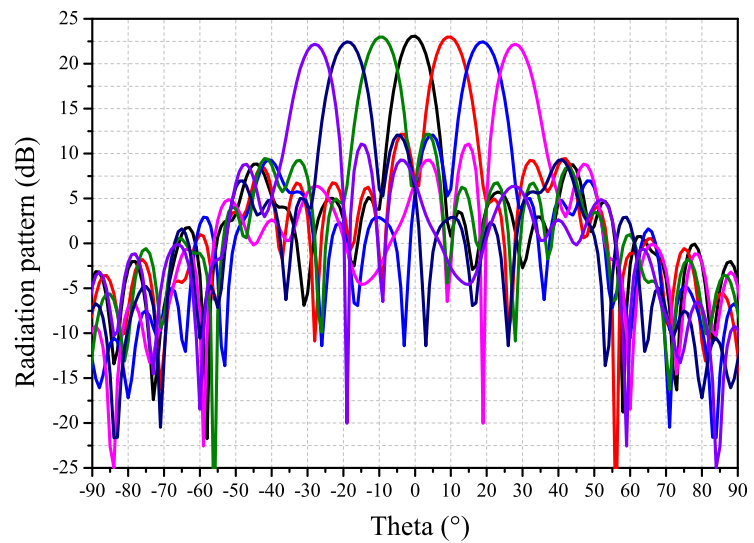

(b)

Figure 11. Simulated radiation patterns at $11.8 \mathrm{GHz}$ with main beam tilted from $-30^{\circ}$ to $+30^{\circ}$ in (a) E-plane and (b) H-plane.

Figure 10 illustrates the phase distributions to steer the main beam directions along the theta angle in both E-plane $\left(\phi=0^{\circ}\right)$ and H-plane $\left(\phi=90^{\circ}\right)$. All phase distributions are constructed using three phase states. For the transmitarray, the elements with all diodes being OFF are used to provide the phase state $0^{\circ}$. The elements with first pair of diodes in ON state are used to provide the second phase state $\left(120^{\circ}\right)$. The elements with the second pair of diodes being ON provide the third phase states $\left(240^{\circ}\right)$. The scanning performance of the transmitarray has been evaluated by simulations. The radiation patterns at $11.5 \mathrm{GHz}$ for different main beam directions varying from $-30^{\circ}$ to $+30^{\circ}$ for both E-plane and H-plane are shown in Figure 11. It can be seen that the main beam changes consistently with phase arrangement and low side lobes are obtained. The maximum directivity is $23.9 \mathrm{dBi}$ when the main beam is at broadside direction while the scan loss reaches $1.6 \mathrm{~dB}$ and $2.9 \mathrm{~dB}$ for beams tilted at $\pm 30^{\circ}$ in $\mathrm{H}$ - and E- planes, respectively. In comparison with the 1-bit element for reconfigurable transmitarray in [12] where the maximum directivity is reported with $21 \mathrm{dBi}$, the transmitarray validated in this paper provides higher efficiency while the size and the number of elements are identical.

\section{Conclusion}

The three-phase-state element for reconfigurable transmitarray has been presented in this paper. Both simulation and measurement results validated good phase shifting capability and a wide $-3 \mathrm{~dB}$ transmission bandwidth. While the prototype is still passive, where the ideal metallic strips are used as p-i-n diodes, simulation results indicated that the fully populated reconfigurable transmitarray can provide a wide scan angle with low scan loss. Further study and implementation of real $\mathrm{p}$-i-n diodes will be deployed in an electronically tunable version of the transmitarray.

\section{ACKNOWLEDGMENT}

This research is funded by the Vietnam National Foundation for Science and Technology Development (NAFOSTED) under grant number 102.01-2016.35.

\section{REFERENCES}

[1] C. G. M. Ryan, M. R. Chaharmir, J. R. B. J. Shaker, J. R. Bray, Y. M. M. Antar, and A. Ittipiboon, "A wideband transmitarray using dual-resonant double square rings," IEEE Transactions on Antennas and Propagation, vol. 58, no. 5, pp. 1486-1493, 2010.

[2] G. Liu, H.-j. Wang, J.-s. Jiang, F. Xue, and M. Yi, “A highefficiency transmitarray antenna using double split ring slot elements," IEEE Antennas and Wireless Propagation Letters, vol. 14, pp. 1415-1418, 2015.

[3] C. Tian, Y.-C. Jiao, G. Zhao, and H. Wang, "A wideband transmitarray using triple-layer elements combined with cross slots and double square rings," IEEE Antennas and Wireless Propagation Letters, vol. 16, pp. 1561-1564, 2017.

[4] B. Rahmati and H. R. Hassani, "High-efficient wideband slot transmitarray antenna," IEEE Transactions on Antennas and Propagation, vol. 63, no. 11, pp. 5149-5155, 2015.

[5] T. Nguyen, B. D. Nguyen, V.-S. Tran, M. Linh, and L. H. Trinh, "Wideband unit-cell for linearly polarized X-band transmitarray applications," in 2018 IEEE International Conference on Advanced Technologies for Communications (ATC), 2018, pp. 125-128.

[6] P. Padilla, A. Munoz-Acevedo, M. Sierra-Castaner, and M. Sierra-Perez, "Electronically reconfigurable transmitarray at $\mathrm{Ku}$ band for microwave applications," IEEE Transactions on Antennas and Propagation, vol. 58, no. 8, pp. 2571-2579, 2010.

[7] J. Y. Lau and S. V. Hum, "A wideband reconfigurable transmitarray element," IEEE Transactions on Antennas and Propagation, vol. 60, no. 3, pp. 1303-1311, 2011.

[8] L. Boccia, I. Russo, G. Amendola, and G. Di Massa, "Multilayer antenna-filter antenna for beam-steering transmit-array applications," IEEE transactions on microwave theory and techniques, vol. 60, no. 7, pp. 2287-2300, 2012.

[9] C.-C. Cheng, B. Lakshminarayanan, and A. AbbaspourTamijani, "A programmable lens-array antenna with monolithically integrated MEMS switches," IEEE Transactions on Microwave Theory and Techniques, vol. 57, no. 8, pp. 18741884, 2009.

[10] L. Di Palma, A. Clemente, L. Dussopt, R. Sauleau, P. Potier, and P. Pouliguen, "1-bit reconfigurable unit cell for Ka-band transmitarrays," IEEE Antennas and Wireless Propagation Letters, vol. 15, pp. 560-563, 2015. 
[11] A. Clemente, L. Dussopt, R. Sauleau, P. Potier, and P. Pouliguen, "1-Bit reconfigurable unit cell based on PIN diodes for transmit-array applications in X-band," IEEE Transactions on Antennas and Propagation, vol. 60, no. 5, pp. 2260-2269, 2012.

[12] B. D. Nguyen and C. Pichot, "Unit-cell loaded with PIN diodes for 1-bit linearly polarized reconfigurable transmitarrays," IEEE Antennas and Wireless Propagation Letters, vol. 18 , no. 1, pp. 98-102, 2018.

[13] F. Diaby, A. Clemente, L. Di Palma, L. Dussopt, K. Pham, E. Fourn, and R. Sauleau, "Linearly-polarized electronically reconfigurable transmitarray antenna with 2-bit phase resolution in Ka-band," in 2017 IEEE International Conference on Electromagnetics in Advanced Applications (ICEAA), 2017, pp. 1295-1298.

[14] B. D. Nguyen and M. T. Nguyen, "Three-bit unit-cell with low profile for X-band linearly polarized transmitarrays." Applied Computational Electromagnetics Society Journal, vol. 38, no. 9, 2019.

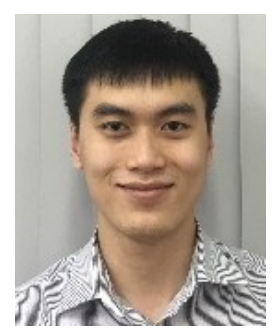

Nguyen Minh Thien was born in Vietnam in 1995. He received his Bachelor of Engineering in Electrical Engineering from the International University, Ho Chi Minh City in 2017. He is currently pursuing a Master program in the School of Electrical Engineering, International University. His research interests mainly focus on design high gain antenna array, unit-cell design for passive reflectarray, transmitarrays, electronically reconfigurable transmitarray.

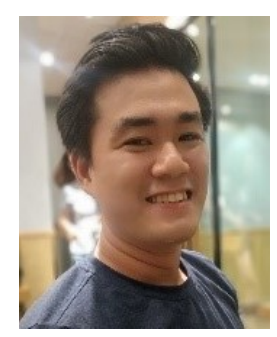

Nguyen Huu Minh was born in Vietnam in 1992. He received his Bachelor of Engineering in Electrical Engineering from the International University, Ho Chi Minh City in 2019. He is currently working as a hardware engineer for Homa Techs Inc. His interests mainly focus on passive and active transmitarrays, PCB antenna design.

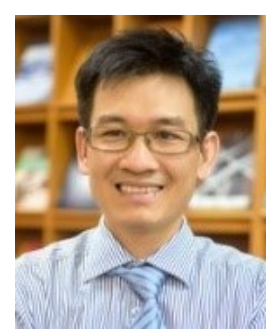

Nguyen Binh Duong was born in Vietnam in 1976. He received the B.S. degree in electronic and electrical engineering from Ho Chi Minh University of Technologies, Ho Chi Minh, Vietnam, in 2000 and the M.S. and Ph.D. degrees in electronic engineering from the University of NiceSophia Antipolis, France, in 2001 and 2006 respectively. From 2001 to 2006, he was as a Researcher at the Laboratoire d'Electronique d'Antennes et Telecommunication, University of Nice-Sophia Antipolis, France. His research interests focus on millimeter antenna, reflector, reflectarray and FSS. 